\title{
Continuous Dependence for Two Implicit Kirk-Type Algorithms in General Hyperbolic Spaces
}

\author{
K. Rauf, ${ }^{1}$ O. T. Wahab, ${ }^{2}$ and S. M. Alata ${ }^{1}$ \\ ${ }^{1}$ Department of Mathematics, University of Ilorin, Ilorin, Nigeria \\ ${ }^{2}$ Department of Statistics and Mathematical Sciences, Kwara State University, Malete, Nigeria \\ Correspondence should be addressed to K. Rauf; krauf@unilorin.edu.ng
}

Received 17 January 2017; Accepted 4 May 2017; Published 21 June 2017

Academic Editor: Hichem Ben-El-Mechaiekh

Copyright (c) $2017 \mathrm{~K}$. Rauf et al. This is an open access article distributed under the Creative Commons Attribution License, which permits unrestricted use, distribution, and reproduction in any medium, provided the original work is properly cited.

This paper aims to study extensively some results concerning continuous dependence for implicit Kirk-Mann and implicit KirkIshikawa iterations. In order to equipoise the formation of these algorithms, we introduce a general hyperbolic space which is no doubt a free associate of some known hyperbolic spaces. The present results are extension of other results and they can be used in many applications.

\section{Introduction}

In [1], Kohlenbach defined hyperbolic space in his paper titled "Some Logical Metatheorems with Applications in Functional Analysis, Transactions of the American Mathematical Society, Vol. 357, 89-128." He combined a metric space $(X, d)$ and a convexity mapping $W: X^{2} \times[0,1] \rightarrow X$ which satisfy

(W1) $d(z, W(x, y, \lambda)) \leq(1-\lambda) d(z, x)+\lambda d(z, y)$,

(W2) $d\left(W\left(x, y, \lambda_{1}\right), W\left(x, y, \lambda_{2}\right)\right)=\left|\lambda_{1}-\lambda_{2}\right| d(x, y)$,

(W3) $W(x, y, \lambda)=W(x, y, 1-\lambda)$,

(W4) $d(W(x, z, \lambda), W(y, w, \lambda)) \leq(1-\lambda) d(x, y)+\lambda d(z, w)$,

for all $x, y, z, w \in X$ and $\lambda, \lambda_{1}, \lambda_{2} \in[0,1]$.

Due to the rich geometric properties of this space, a large amount of results have been published on hyperbolic spaces such as [2-4]. It is observed that conditions (W1)-(W4) can only be fulfilled for two or three distinct points. So, to balance up the proportions of the space against the iterative processes in question, we introduce a general notion of the hyperbolic space. Firstly, we define the following.
Definition 1. Let $(X, d)$ be a metric space. A mapping $W: X^{k} \times$ $[0,1]^{k} \rightarrow X$ is called a generalized convex structure on $X$ if for each $x_{i} \in X$ and $\lambda_{i} \in[0,1]$

$$
d\left(q, W\left(x_{1}, x_{2}, \ldots, x_{k} ; \lambda_{1}, \lambda_{2}, \ldots, \lambda_{k}\right)\right) \leq \sum_{i=1}^{k} \lambda_{i} d\left(q, x_{i}\right)
$$

holds for $q \in X$ and $\sum_{i=1}^{k} \lambda_{i}=1$. The metric space $(X, d)$ together with a generalized convex structure $W$ is called a generalized convex metric space.

By letting $k=3$ and $k=2$, we retrieve the convex metric space in $[5,6]$, respectively.

We now give the following definition.

Definition 2. Let $(X, d)$ be a metric space and $W: X^{k} \times$ $[0,1]^{k} \rightarrow X$. A general hyperbolic space is a metric space $(X, d)$ associated with the mapping $W$ and it satisfies the following:

$(\mathrm{GW} 1) d\left(y, W\left(x_{1}, x_{2}, \ldots, x_{k} ; \lambda_{1}, \lambda_{2}, \ldots, \lambda_{k}\right)\right) \leq \sum_{i=1}^{k} \lambda_{i} d(y$, $\left.x_{i}\right)$,

$(\mathrm{GW} 2) d\left(W\left(x_{1}, x_{2}, \ldots, x_{k} ;[0,1]_{\lambda}^{k}\right), W\left(x_{1}, x_{2}, \ldots, x_{k} ;[0,1]_{\mu}^{k}\right)\right)=$ $\sum_{i=1}^{k-1}\left|\lambda_{i}-\mu_{i}\right| d\left(x_{i}, x_{i+1}\right)$

(GW3) $W\left(x_{1}, x_{2}, \ldots, x_{k} ; \lambda_{1}, \lambda_{2}, \ldots, \lambda_{k}\right)=W\left(x_{k}, \ldots, x_{2}, x_{1}\right.$; $\left.1-\lambda_{1}, 1-\lambda_{2}, \ldots, 1-\lambda_{k}\right)$, 
(GW4) $d\left(W\left(x_{1}, x_{2}, \ldots, x_{k} ; \lambda_{1}, \lambda_{2}, \ldots, \lambda_{k}\right), W\left(y_{1}, y_{2}, \ldots, y_{k}\right.\right.$; $\left.\left.\lambda_{1}, \lambda_{2}, \ldots, \lambda_{k}\right)\right) \leq \sum_{i=1}^{k} \lambda_{i} d\left(x_{i}, y_{i}\right)$,

where $[0,1]_{\lambda}^{k}=\lambda_{1}, \lambda_{2}, \ldots, \lambda_{k}$, for each $\lambda_{i} \in[0,1]$ and $x_{i}, y_{i}, y \in X, i=1(1) k$.

It is easily seen that Definition 2 is hyperbolic space when $k=2$.

We note here that every general hyperbolic space is a generalized convex metric space, but the converse in some cases is not necessarily true.

For example, let $X^{k}=\mathbb{R}^{k}$ be endowed with the metric $d(\underline{x}, \underline{y})=\sum_{i}^{k}\left(\left|x_{i}-y_{i}\right| /\left(1+\left|x_{i}-y_{i}\right|\right)\right)$ and $W\left(x_{1}, x_{2}\right.$, $\left.\ldots, x_{k} ; \lambda_{1}, \lambda_{2}, \ldots, \lambda_{k}\right)=\sum \lambda_{i} x_{i}$, for $\underline{x}, y \in \mathbb{R}^{k}$; then, metric $d$ on $\mathbb{R}^{k}$ associated with $W$ is a generalized convex metric space but it does not satisfy all the conditions (GW1)-(GW4).

Two hybrid Kirk-type schemes, namely, Kirk-Mann and Kirk-Ishikawa iterations, were first introduced in normed linear space as appeared in [7]. Remarkable results have been investigated to date for more cases of Kirk-type schemes; see [8-11]. Recently in [12], the implicit Kirk-type schemes were introduced in Banach space for a contractive-type operator and it was also remarkable.

However, there are few or no emphases on the data dependence of the Kirk-type schemes. Hence, this paper aims to study closely the continuous contingency of two Kirk-type schemes in [12], namely, implicit Kirk-Mann and implicit Kirk-Ishikawa iterations in a general hyperbolic space. To do this, a certain approximate operator (say $S$ ) of $T$ is used to access the same source as $T$ in such a way that $d(T x, S x) \leq \eta$ for all $x \in X$ and $\eta>0$.

We shall employ the class of quasi-contractive operator:

$$
\begin{aligned}
& d(T x, T y) \leq a d(x, y)+\epsilon d(x, T x) \\
& \qquad \text { for } x, y \in X, \epsilon \geq 0, a \in(0,1)
\end{aligned}
$$

in [13] to prove the following lemma.

Lemma 3. Let $(X, d)$ be a metric space and let $T: X \rightarrow X$ be a map satisfying (2). Then, for all $k \in \mathbb{N}$ and $\epsilon \geq 0$

$$
d\left(T^{k} x, T^{k} y\right) \leq \sum_{i=1}^{k}\left(\begin{array}{l}
k \\
i
\end{array}\right) a^{k-i} \epsilon^{i} d\left(x, T^{i} x\right)+a^{k} d(x, y)
$$

for all $x, y \in X$ and $a \in(0,1)$.

Proof. Let $T$ be an operator satisfying (2); we claim that $T^{k} x$ also satisfies (2).

Then,

$$
\begin{aligned}
d\left(T^{k} x, T^{k} y\right) \leq & \epsilon d\left(x, T^{k} x\right)+a d\left(T^{k-1} x, T^{k-1} y\right) \\
\leq & \epsilon d\left(x, T^{k} x\right)+a \epsilon d\left(x, T^{k-1} x\right) \\
& +a^{2} d\left(T^{k-2} x, T^{k-2} y\right) \leq \cdots
\end{aligned}
$$

$$
\leq \sum_{i=1}^{k}\left(\begin{array}{l}
k \\
i
\end{array}\right) a^{k-i} \epsilon^{i} d\left(x, T^{i} x\right)+a^{k} d(x, y)
$$

for each $a^{k} \in(0,1)$ and $\epsilon^{i} \geq 0$. Thus, $T^{k} x$ satisfies (3).

The converse of Lemma 3 is not true for $k>1$. Hence, condition (3) is more general than (2).

Lemma 4 (see [14]). Let $\left\{a_{n}\right\}_{n=0}^{\infty}$ be a nonnegative sequence for which there exists $n_{0} \in \mathbb{N}$ such that, for all $n \geq n_{0}$, one has the following inequality:

$$
a_{n+1} \leq\left(1-r_{n}\right) a_{n}+r_{n} t_{n}
$$

where $r_{n} \in(0,1)$, for all $n \in \mathbb{N}, \sum_{n=1}^{\infty} r_{n}=\infty$, and $t_{n} \geq 0$ for $n \in \mathbb{N}$. Then

$$
0 \leq \lim _{n \rightarrow \infty} \sup a_{n} \leq \lim _{n \rightarrow \infty} \sup t_{n}
$$

\section{Main Results}

We present the results for implicit Kirk-Mann and implicit Kirk-Ishikawa iterations using condition (3) and noting that both iterations converge strongly to a fixed point $p \in F_{T}$ as proved in [12].

Theorem 5. Let $K$ be a closed subset of a general hyperbolic space $(X, d, W)$ and let $T, S: K \rightarrow K$ be maps satisfying (3), where $S$ is an approximate operator of $T$. Let $\left\{x_{n}\right\},\left\{u_{n}\right\} \subset K$ be two iterative sequences associated with $T$, respectively, to $S$ given as follows: for $x_{0}, u_{0} \in X$

$$
\begin{aligned}
& x_{n}=W\left(x_{n-1}, T x_{n}, T^{2} x_{n}, \ldots, T^{k} x_{n} ; \alpha_{n, 0}, \alpha_{n, 1}, \alpha_{n, 2}, \ldots,\right. \\
& \left.\alpha_{n, k}\right) ; \\
& \sum_{i=0}^{k} \alpha_{n, i}=1, \quad n \geq 1, \\
& u_{n}=W\left(u_{n-1}, S u_{n}, S^{2} u_{n}, \ldots, S^{k} u_{n} ; \alpha_{n, 0}, \alpha_{n, 1}, \alpha_{n, 2}, \ldots, \alpha_{n, k}\right) ; \\
& \sum_{i=0}^{k} \alpha_{n, i}=1,
\end{aligned}
$$

$$
n \geq 1 \text {, }
$$

where $\alpha_{n, i}, \beta_{n, i}$ are sequences in $[0,1]$, for $i=0,1,2, \ldots, k, k \in$ $\mathbb{N}$ with $\sum\left(1-\alpha_{n, 0}\right)=\infty$.

If $p \in F_{T}, q \in F_{S}$ and $\eta>0$, then

$$
d(p, q) \leq \frac{\eta}{(1-a)^{2}}
$$


Proof. Let $x_{0}, u_{0} \in X, p \in F_{T}$, and $q \in F_{S}$. By using (GW1)-(GW4), (7), (8), and (3), we get

$$
\begin{aligned}
& d\left(x_{n}, u_{n}\right)=d\left(W \left(x_{n-1}, T x_{n}, T^{2} x_{n}, \ldots, T^{k} x_{n} ; \alpha_{n, 0}, \alpha_{n, 1},\right.\right. \\
& \left.\alpha_{n, 2}, \ldots, \alpha_{n, k}\right), W\left(u_{n-1}, S u_{n}, S^{2} u_{n}, \ldots, S^{k} u_{n} ; \alpha_{n, 0}, \alpha_{n, 1},\right. \\
& \left.\left.\alpha_{n, 2}, \ldots, \alpha_{n, k}\right)\right) \leq \alpha_{n, 0} d\left(x_{n-1}, u_{n-1}\right) \\
& +\sum_{i=1}^{k} \alpha_{n, i}\left(d\left(T^{i} x_{n}, S^{i} x_{n}\right)+d\left(S^{i} x_{n}, S^{i} u_{n}\right)\right) \\
& \leq \alpha_{n, 0} d\left(x_{n-1}, u_{n-1}\right)+\sum_{i=1}^{k} \alpha_{n, i}[\eta \\
& \left.+\sum_{i=1}^{k}\left(\begin{array}{l}
k \\
i
\end{array}\right) a^{k-i} \epsilon^{i} d\left(x_{n}, S^{i} x_{n}\right)+a^{k} d\left(x_{n}, u_{n}\right)\right]
\end{aligned}
$$

which implies

$$
\begin{aligned}
& d\left(x_{n}, u_{n}\right) \\
& \leq \frac{\alpha_{n, 0}}{1-\sum_{i=1}^{k} \alpha_{n, i} a^{i}} d\left(x_{n-1}, u_{n-1}\right) \\
& \quad+\frac{\sum_{i=1}^{k} \alpha_{n, i}}{1-\sum_{i=1}^{k} \alpha_{n, i} a^{i}}\left(\eta+\sum_{i=1}^{k}\left(\begin{array}{c}
k \\
i
\end{array}\right) a^{k-i} \epsilon^{i} d\left(x_{n}, S^{i} x_{n}\right)\right)
\end{aligned}
$$

$$
\left.+\sum_{i=1}^{k}\left(\begin{array}{l}
k \\
i
\end{array}\right) a^{k-i} \epsilon^{i} d\left(x_{n}, S^{i} x_{n}\right)\right) .
$$

Let $Q_{n}=\alpha_{n, 0} /\left(1-\left(1-\alpha_{n, 0}\right) a\right)$; then

$$
\begin{aligned}
1-Q_{n} & =\frac{1-\left(\alpha_{n, 0}+\left(1-\alpha_{n, 0}\right) a\right)}{1-\left(1-\alpha_{n, 0}\right) a} \\
& \geq 1-\left(\alpha_{n, 0}+\left(1-\alpha_{n, 0}\right) a\right) .
\end{aligned}
$$

Hence, we have

$$
Q_{n} \leq \alpha_{n, 0}+\left(1-\alpha_{n, 0}\right) a=1-(1-a)\left(1-\alpha_{n, 0}\right) .
$$

Using (14) and the fact that $1-a \leq 1-\left(1-\alpha_{n, 0}\right) a$ then (12) becomes

$$
\begin{gathered}
d\left(x_{n}, u_{n}\right) \leq\left[1-(1-a)\left(1-\alpha_{n, 0}\right)\right] d\left(x_{n-1}, u_{n-1}\right) \\
+\frac{(1-a)\left(1-\alpha_{n, 0}\right)}{(1-a)^{2}}\left(\eta+\sum_{i=1}^{k}\left(\begin{array}{c}
k \\
i
\end{array}\right) a^{k-i} \epsilon^{i} d\left(x_{n}, S^{i} x_{n}\right)\right) .
\end{gathered}
$$

By letting $a_{n}=d\left(x_{n}, u_{n}\right), r_{n}=(1-a)\left(1-\alpha_{n, 0}\right)$, and $t_{n}=$ $\left(1 /(1-a)^{2}\right)\left(\eta+\sum_{i=1}^{k}\left(\begin{array}{c}k \\ i\end{array}\right) a^{k-i} \epsilon^{i} d\left(x_{n}, S^{i} x_{n}\right)\right)$ in (15).
Thus, by Lemma 4, inequality (15) becomes

$$
\begin{aligned}
& \lim _{n \rightarrow \infty} d\left(x_{n}, u_{n}\right) \\
& \quad \leq \frac{1}{(1-a)^{2}}\left(\eta+\sum_{i=1}^{k}\left(\begin{array}{l}
k \\
i
\end{array}\right) a^{k-i} \epsilon^{i} \lim _{n \rightarrow \infty} d\left(x_{n}, S^{i} x_{n}\right)\right) .
\end{aligned}
$$

for

$$
\begin{aligned}
0 & \leq d\left(x_{n}, S^{i} x_{n}\right) \leq d\left(x_{n}, p\right)+\left(s^{i} p, S^{i} x_{n}\right) \\
& \leq\left(1+a^{i}\right) d\left(x_{n}, p\right) \longrightarrow 0, \text { as } n \longrightarrow \infty
\end{aligned}
$$

Therefore,

$$
d(p, q) \leq \frac{\eta}{(1-a)^{2}}
$$

Theorem 6. Let $K \subset(X, d, W)$ and $T, S: K \rightarrow K$ be two maps satisfying (3), where $S$ is an approximate operator of $T$. Let $\left\{x_{n}\right\},\left\{u_{n}\right\}$ be two implicit Kirk-Ishikawa iterative sequences associated with $T$, respectively, to $S$ given as follows: for $x_{0}, u_{0} \in$ $X$

$$
\begin{aligned}
& x_{n}=W\left(y_{n-1}, T x_{n}, T^{2} x_{n}, \ldots, T^{k} x_{n} ; \alpha_{n, 0}, \alpha_{n, 1}, \alpha_{n, 2}, \ldots,\right. \\
& \left.\alpha_{n, k}\right) ; \\
& \sum_{i=0}^{k} \alpha_{n, i}=1, \\
& y_{n-1}=W\left(x_{n-1}, T y_{n-1}, T^{2} y_{n-1}, \ldots, T^{s} y_{n-1} ; \beta_{n, 0}, \beta_{n, 1},\right. \\
& \left.\quad \beta_{n, 2}, \ldots, \beta_{n, s}\right) ; \\
& \sum_{i=0}^{s} \beta_{n, i}=1,
\end{aligned}
$$

$$
n \geq 1 \text {, }
$$$$
u_{n}=W\left(v_{n-1}, S u_{n}, S^{2} u_{n}, \ldots, S^{k} u_{n} ; \alpha_{n, 0}, \alpha_{n, 1}, \alpha_{n, 2}, \ldots,\right.
$$$$
\left.\alpha_{n, k}\right) \text {; }
$$$$
\sum_{i=0}^{k} \alpha_{n, i}=1
$$$$
v_{n-1}=W\left(u_{n-1}, S v_{n-1}, S^{2} v_{n-1}, \ldots, S^{s} v_{n-1} ; \beta_{n, 0}, \beta_{n, 1}, \beta_{n, 2}\right. \text {, }
$$

$\left.\ldots, \beta_{n, s}\right)$;

$\sum_{i=0}^{s} \beta_{n, i}=1$ 
where $\alpha_{n, i_{k}}, \beta_{n, i_{s}}$ are sequences in $[0,1]$, for $i_{k}=0(1) k ; i_{s}=$ $0(1) s ; k$ and $s$ are fixed integers such that $k \geq s$ with $\sum(1-$ $\left.\alpha_{n, 0}\right)=\infty$. Assume that $p \in F_{T}, q \in F_{S}$, and $\eta>0$; then

$$
d(p, q) \leq \frac{2 \eta}{(1-a)^{2}}
$$

Proof. Let $x_{0}, u_{0} \in X$. By taking $x_{n}$ of (19) and $u_{n}$ of (20) using conditions (GW1)-(GW4) and (3), we obtain

$$
\begin{aligned}
& d\left(x_{n}, u_{n}\right) \leq \frac{\alpha_{n, 0}}{1-\sum_{i_{k}=1}^{k} \alpha_{n, i_{k}} a^{i_{k}}} d\left(y_{n-1}, v_{n-1}\right) \\
& +\frac{\sum_{i_{k}=1}^{k} \alpha_{n, i_{k}}}{1-\sum_{i_{k}=1}^{k} \alpha_{n, i_{k}} a^{i_{k}}}(\eta \\
& \left.+\sum_{i_{k}=1}^{k}\left(\begin{array}{c}
k \\
i_{k}
\end{array}\right) a^{k-i_{k}} \epsilon^{i_{k}} d\left(x_{n}, S^{i_{k}} x_{n}\right)\right) .
\end{aligned}
$$

Similarly, $y_{n-1}$ of (19) and $v_{n-1}$ of (20) give

$$
\begin{aligned}
& d\left(y_{n-1}, v_{n-1}\right) \leq \frac{\beta_{n, 0}}{1-\sum_{i_{s}=1}^{s} \beta_{n, i_{s}} a_{s}^{i_{s}}} d\left(x_{n-1}, u_{n-1}\right) \\
& +\frac{\sum_{i_{s}=1}^{s} \beta_{n, i_{s}}}{1-\sum_{i_{s}=1}^{s} \beta_{n, i_{s}} a^{i_{s}}}(\eta \\
& \left.+\sum_{i_{s}=1}^{s}\left(\begin{array}{c}
s \\
i_{s}
\end{array}\right) a^{s-i_{s}} e^{i_{s}} d\left(x_{n}, S^{i_{s}} x_{n}\right)\right)
\end{aligned}
$$

By combining (22) and (23), we have

$$
\begin{aligned}
& d\left(x_{n}, u_{n}\right) \\
& \leq \frac{\alpha_{n, 0}}{1-\sum_{i_{k}=1}^{k} \alpha_{n, i_{k}} a^{i_{k}}}\left[\frac { \beta _ { n , 0 } } { 1 - \sum _ { i _ { s } = 1 } ^ { s } \beta _ { n , i _ { s } } a ^ { i _ { s } } } d \left(x_{n-1},\right.\right. \\
& \left.u_{n-1}\right)+\frac{\sum_{i_{s}=1}^{s} \beta_{n, i_{s}}}{1-\sum_{i_{s}=1}^{s} \beta_{n, i_{s}} a^{i_{s}}} \\
& \left.\quad \times\left(\eta+\sum_{i_{s}=1}^{s}\left(\begin{array}{c}
s \\
i_{s}
\end{array}\right) a^{s-i_{s}} \epsilon^{i_{s}} d\left(x_{n}, S^{i_{s}} x_{n}\right)\right)\right] \\
& +\frac{\sum_{i_{k}=1}^{k} \alpha_{n, i_{k}}}{1-\sum_{i_{k}=1}^{k} \alpha_{n, i_{k}} a^{i_{k}}}(\eta \\
& \left.\quad+\sum_{i_{k}=1}^{k}\left(\begin{array}{c}
k \\
i_{k}
\end{array}\right) a^{k-i_{k}} \epsilon^{i_{k}} d\left(x_{n}, S^{i_{k}} x_{n}\right)\right) .
\end{aligned}
$$

This is further reduced to

$$
\begin{aligned}
& d\left(x_{n}, u_{n}\right) \\
& \leq \frac{\alpha_{n, 0} \beta_{n, 0}}{\left[1-\left(1-\alpha_{n, 0}\right) a\right]\left[1-\left(1-\beta_{n, 0}\right) a\right]} d\left(x_{n-1},\right. \\
& \left.u_{n-1}\right)+\frac{\alpha_{n, 0}\left(1-\beta_{n, 0}\right)}{\left[1-\left(1-\alpha_{n, 0}\right) a\right]\left[1-\left(1-\beta_{n, 0}\right) a\right]} \times(\eta \\
& \left.+\sum_{i_{s}=1}^{s}\left(\begin{array}{c}
s \\
i_{s}
\end{array}\right) a^{s-i_{s}} \epsilon^{i_{s}} d\left(x_{n}, S^{i_{s}} x_{n}\right)\right) \\
& +\frac{1-\alpha_{n, 0}}{1-\left(1-\alpha_{n, 0}\right) a}\left(\eta+\sum_{i_{k}=1}^{k}\left(\begin{array}{l}
k \\
i_{k}
\end{array}\right)\right. \\
& \left.\cdot a^{k-i_{k}} \epsilon^{i_{k}} d\left(x_{n}, S^{i_{k}} x_{n}\right)\right) .
\end{aligned}
$$

Using the ansatz prescribed in (14), we get

$$
\begin{aligned}
& +\frac{(1-a)\left(1-\alpha_{n, 0}\right)}{(1-a)^{2}} \times(2 \eta \\
& +\sum_{i_{s}=1}^{s}\left(\begin{array}{l}
s \\
i_{s}
\end{array}\right) a^{s-i_{s}} \epsilon^{i_{s}} d\left(x_{n}, S^{i_{s}} x_{n}\right) \\
& \left.+\sum_{i_{k}=1}^{k}\left(\begin{array}{l}
k \\
i_{k}
\end{array}\right) a^{k-i_{k}} \epsilon^{i_{k}} d\left(x_{n}, S^{i_{k}} x_{n}\right)\right) .
\end{aligned}
$$$$
d\left(x_{n}, u_{n}\right) \leq\left[1-(1-a)\left(1-\alpha_{n, 0}\right)\right] d\left(x_{n-1}, u_{n-1}\right)
$$

Using the condition of Lemma 4, we conclude that

$$
\lim _{n \rightarrow \infty} d\left(x_{n}, u_{n}\right) \longrightarrow d(p, q) \leq \frac{2 \eta}{(1-a)^{2}} .
$$

This following example is adopted from [14].

Example 7. Let $T: \mathbb{R} \rightarrow \mathbb{R}$ be given by

$$
T x= \begin{cases}0 & \text { if } x \in(-\infty, 2] \\ -0.5 & \text { if } x \in(2, \infty)\end{cases}
$$

with the unique fixed point being 0 . Then, $T$ is quasicontractive operator.

Also, consider the map $S: \mathbb{R} \rightarrow \mathbb{R}$,

$$
S x= \begin{cases}1 & \text { if } x \in(-\infty, 2] \\ -1.5 & \text { if } x \in(2, \infty)\end{cases}
$$

with the unique fixed point 1.

Take $\eta$ to be the distance between the two maps as follows:

$$
d(S x, T x) \leq 1, \quad \forall x \in \mathbb{R} .
$$


TABLE 1

\begin{tabular}{lcc}
\hline Number of iterations & Iteration $(7)$ & Iteration (19) \\
\hline 5 & 0.8944272 & 0.9888544 \\
6 & 0.9806270 & 0.9996247 \\
7 & 0.9952716 & 0.9999776 \\
8 & 0.9986151 & 0.9999981 \\
9 & 0.9995384 & 0.9999998 \\
10 & 0.9998303 & 1.0000000 \\
11 & 0.9999326 & 1.0000000 \\
$\vdots$ & $\vdots$ & $\vdots$ \\
21 & 0.9999999 & 1.0000000 \\
22 & 0.9999999 & 1.0000000 \\
23 & 1.0000000 & 1.0000000 \\
\hline
\end{tabular}

Let $x_{0}=u_{0}=0$ be the initial datum, $\alpha_{n, 0}=\beta_{n, 0}=1-2 / \sqrt{n}$, and $\alpha_{n, i}=\beta_{n, i}=1 / \sqrt{n}$ for $n \geq 5, i=1,2$. Note that $\alpha_{n, i}=$ $\beta_{n, i}=0$ for $n=1(1) 4$.

With the aid of MATLAB program, the computational results for the iterations (7) and (19) of operator $S$ are presented in Table 1 with stopping criterion $1 e-8$.

In Table 1, both iterations (7) and (19) converge to the same fixed point 1 . This implies that, for each of the iterations, the distance between the fixed point of $S$ and the fixed point of $T$ is 1 . In fact, this result can also be verified without computing the operator $S$ by using Theorem 5 or Theorem 6 for any choice of $a \in(0,1)$. On the other hand, the result will also be valid if we choose $T$ sufficiently close to $S$.

\section{Concluding Remarks}

These results exhibit sufficient conditions under which approximate fixed points depend continuously on parameters. In fact, the above two results show that $d(p, q) \rightarrow 0$ as $\eta \rightarrow 0$, which is quite remarkable. Also observe there is a tiein between Theorems 5 and 6 in the following order:

$$
d(p, q) \leq \frac{\eta}{(1-a)^{2}} \leq \frac{2 \eta}{(1-a)^{2}} .
$$

Thus, for any case of $k=1,2$, we have

$$
\sup \left\{d(p, q): d(p, q) \leq \frac{k \eta}{(1-a)^{2}}\right\}, \quad \text { for each } k \text {. }
$$

In Example 7 above, $\eta=1$ is chosen, but for higher $k$, it is suitable to choose $\eta=1 / k$.

\section{Disclosure}

The authors agreed to be accountable for all aspects of the work in ensuring that questions related to the accuracy or integrity of any part of the work are appropriately investigated and resolved.

\section{Conflicts of Interest}

Authors hereby declare that there are no conflicts of interest.

\section{References}

[1] U. Kohlenbach, "Some logical metatheorems with applications in functional analysis," Transactions of the American Mathematical Society, vol. 357, no. 1, pp. 89-128, 2005.

[2] A. R. Khan, H. Fukhar-ud-din, and M. A. A. Khan, "An implicit algorithm for two finite families of nonexpansive maps in hyperbolic spaces," Fixed Point Theory and Applications, vol. 2012, article 54, 12 pages, 2012.

[3] M. A. Khan, H. Fukhar-ud-din, and A. Kalsoom, "Existence and higher arity iteration for total asymptotically nonexpansive mappings in uniformly convex hyperbolic spaces," Fixed Point Theory and Applications, 2016:3, 18 pages, 2016.

[4] L. Zhang and X. Wang, " $\Delta$-convergence for common fixed points of two asymptotically nonexpansive nonself mappings in hyperbolic spaces," International Journal of Mathematical Analysis, vol. 9, no. 5-8, pp. 385-393, 2015.

[5] A. Rafiq and S. Zafar, "On the convergence of implicit Ishikawa iterations with error to a common fixed point of two mappings in convex metric space," General Mathematics, vol. 14, no. 2, pp. 95-108, 2006.

[6] W. Takahashi, "A convexity in metric space and nonexpansive mappings I," Kodai Mathematical Seminar Reports, vol. 22, pp. $142-149,1970$.

[7] M. O. Olatinwo, "Some stability results for two hybrid fixed point iterative algorithms in normed linear space," Matematichki Vesnik, vol. 61, no. 4, pp. 247-256, 2009.

[8] H. Akewe, G. A. Okeke, and A. F. Olayiwola, "Strong convergence and stability of Kirk-multistep-type iterative schemes for contractive-type operators," Fixed Point Theory and Applications, vol. 2014, no. 1, article 45, 2014.

[9] R. Chugh and V. Kumar, "Stability of hybrid fixed point iterative algorithms of Kirk-Noor type in normed linear space for self and nonself operators," International Journal of Contemporary Mathematical Sciences, vol. 7, no. 24, pp. 1165-1184, 2012.

[10] F. Gürsoy, V. Karakaya, and B. E. Rhoades, "Data dependence results of new multi-step and S-iterative schemes for contractive-like operators," Fixed Point Theory and Applications, vol. 2013, article 76, 2013:76, 12 pages, 2013.

[11] N. Hussain, R. Chugh, V. Kumar, and A. Rafiq, "On the rate of convergence of Kirk-type iterative schemes," Journal of Applied Mathematics, vol. 2012, Article ID 526503, 22 pages, 2012. 
[12] O. T. Wahab and K. Rauf, "On faster implicit hybrid Kirkmultistep schemes for contractive-type operators," International Journal of Analysis, vol. 2016, Article ID 3791506, 10 pages, 2016.

[13] V. Berinde, "A convergence theorem for some mean value fixed point iterations procedures," Demonstratio Mathematica, vol. 38, p. 17784, 2005.

[14] Ş. M. Şoltuz and T. Grosan, "Data dependence for Ishikawa iteration when dealing with contractive-like operators," Fixed Point Theory and Applications, vol. 2008, Article ID 242916, 7 pages, 2008. 


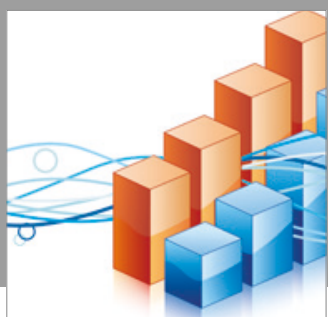

Advances in

Operations Research

vatersals

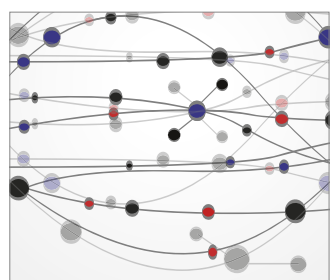

\section{The Scientific} World Journal
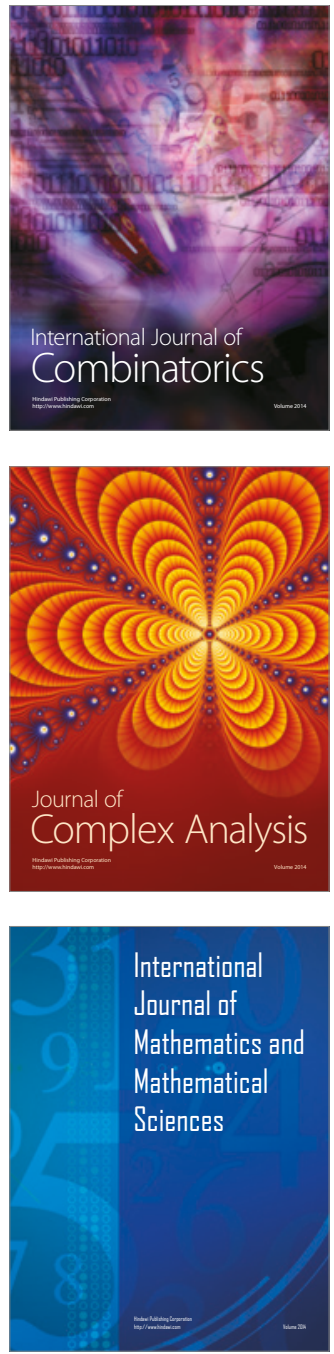
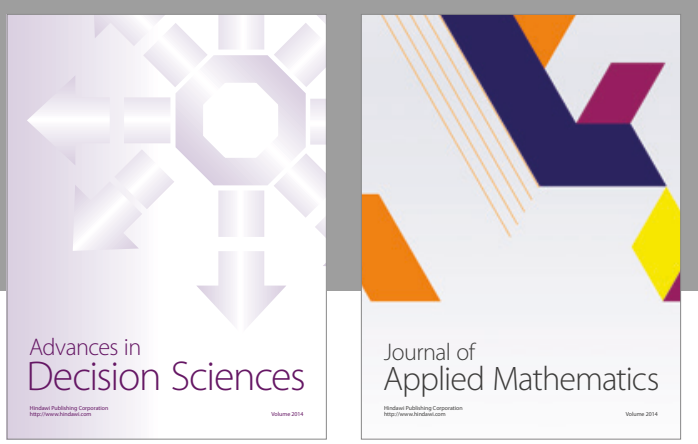

Algebra

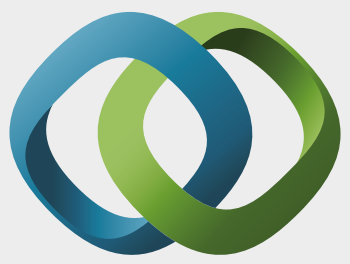

\section{Hindawi}

Submit your manuscripts at

https://www.hindawi.com
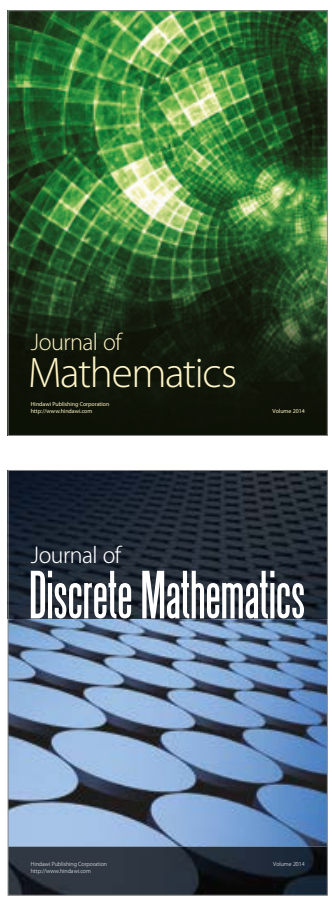

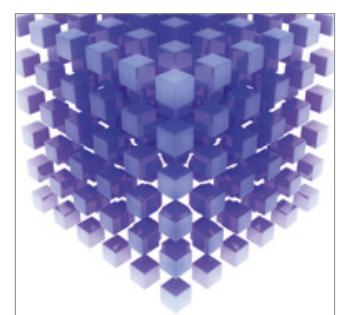

Mathematical Problems in Engineering
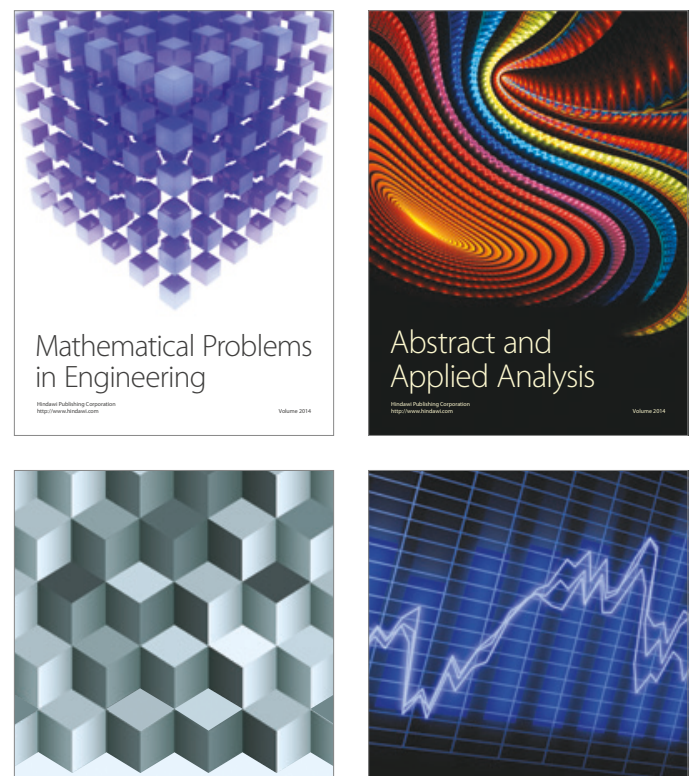

Journal of

Function Spaces

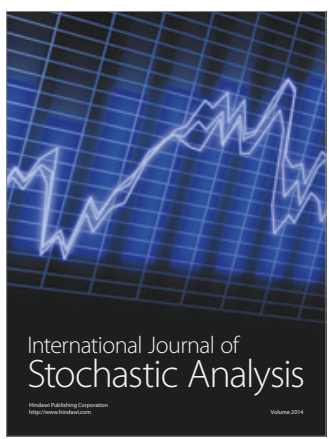

Probability and Statistics
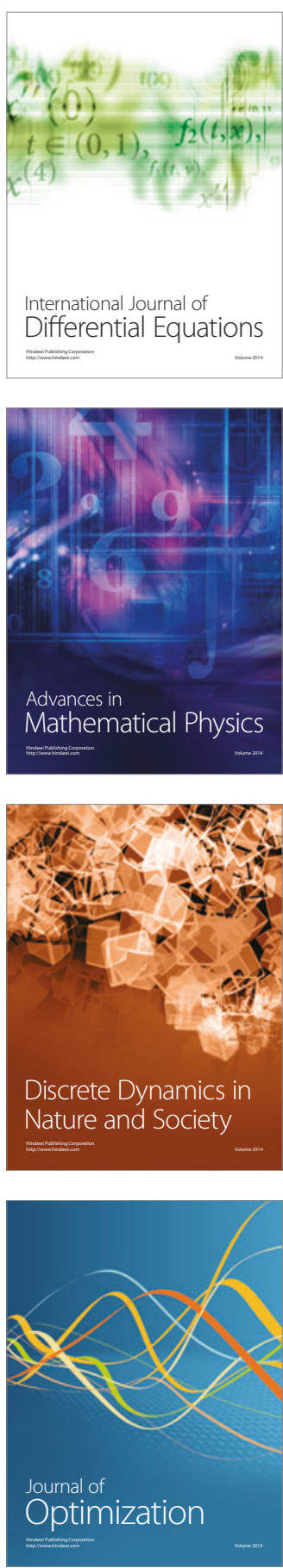